\title{
Evaluation of Differential Evolution Algorithm with Various Mutation Strategies for Clustering Problems
}

\author{
Pyae Pyae Win Cho ${ }^{1}$, Thi Thi Soe Nyunt ${ }^{2}$ \\ ${ }^{1,2}$ University of Computer Studies, Yangon
}

\begin{tabular}{l} 
Article Info \\
\hline Article historys: \\
Received Feb 11, 2020 \\
Revised Dec 15, 2020 \\
Accepted Dec 21, 2020 \\
\hline
\end{tabular}

\section{Keywords:}

Differential Evolution Mutation Strategy

Clustering

\begin{abstract}
Evolutionary Algorithms (EAs) based pattern recognition has emerged as an alternative solution to data analysis problems to enhance the efficiency and accuracy of mining processes. Differential Evolution (DE) is one rival and powerful instance of EAs, and DE has been successfully used for cluster analysis in recent years. Mutation strategy, one of the main processes of DE, uses scaled differences of individuals that are chosen randomly from the population to generate a mutant (trial) vector. The achievement of the DE algorithm for solving optimization problems highly relies on an adopted mutation strategy. In this paper, an empirical study was presented to investigate the effectiveness of six frequently used mutation strategies for solving clustering problems. The experimental tests were conducted on the most widely used data set for EAs based clustering, and the quality of cluster solutions and convergence characteristics of DE variants were evaluated. The obtained results pointed out that the mutation strategies that use the guidance information from the best solution mange to find more stable results whereas the random mutation strategies are able to find high quality solutions with slower convergence rate. This study aims to provide some information and insights to develop better DE mutation schemes for clustering.
\end{abstract}

Copyright () 2020 Institute of Advanced Engineering and Science. All rights reserved.

\section{Corresponding Author:}

Pyae Pyae Win Cho,

University of Computer Studies, Yangon,

No. (4) Main Road, Shwe Pyi Thar Township, Yangon, Myanmar.

Email: pyaepyaewincho@ucsy.edu.mm

\section{INTRODUCTION}

Evolutionary algorithms (EAs), motivated by Darwin's theory of natural selection, have become a powerful way to solve several different optimization problems in various domains [1]. Among them, the Differential Evolution (DE) algorithm is a simple, competent, and robust stochastic search strategy based on population, and it has been successfully used to catch global optimum on high-dimensional continuous problems [2]. Like a standard EA, DE applies evolution processes such as mutation, crossover and selection to transfer from one generation to the next. It differs significantly from other EAs in the fact that the distance between pairs of randomly selected individuals is used to modify the solution, and the selected solution's position guides the track of the search process. To employ the mutation operator in DE, there exist a few different mutation strategies that determine the solution to be modified and the number of different vectors to be used to find the distance for modification [3,4]. The effectiveness of DE heavily depends on the chosen mutation strategy due to different mutation strategies that can guide different tracks toward exploration and exploitation.

In recent years, DE has been widely utilized to solve clustering problems due to its ability to enhance the solution quality. It has been widely used to perform clustering independently [5-7] or incorporate it into the existing clustering approaches [8-10]. Paterlini and Krink described an innovative approach for DE based clustering $[5,6]$. They studied the performance superiority between genetic algorithm (GA), particle swarm optimization (PSO), and DE, and concluded that DE is more suitable for cluster analysis. Some paper proposed the combination of DE with local search approaches to achieve considerably better efficiency [8-10]. 
Nevertheless, it is needed that the comparisons on clustering performance of different mutation strategies for cluster analysis. In this paper, an empirical analysis is presented to compare and examine the performance of DE with different variants of mutation strategy for clustering problems. It is expected that the acquired information insight from the experiments may be useful and helpful to employ optimal mutation strategy for future DE researches in the clustering domain.

In the next section of the paper, a brief explanation of a traditional DE algorithm and different mutation strategies used in DE are presented. The DE based clustering method is explained in Section 3 . In the fourth part, the outcomes of experimental tests are shown, and in Section 5, the paper is completed with a conclusion.

\section{BACKGROUND}

In this section, the basic structure of DE algorithm is firstly described, and then different variants of the mutation strategy used in DE are briefly explained.

\subsection{Differential Evolution Algorithm}

$\mathrm{DE}$ is an innovative heuristic population-based search approach that had been proposed by Storn and Price in 1995. It has become one of the most successful and widely used EAs to solve several real-world continuous global optimization problems in various domains [2, 3]. Like a standard EA, DE maintains a population of individuals that are a sample of candidate solutions to an optimization problem. Hence, an initial population is created through random sampling with uniform distribution at the beginning of the algorithm. And then, DE iteratively performs three consecutive steps (namely mutation, crossover, and selection) until a stopping situation is reached.

Let $X_{i, g}=\left\{x_{i, g}^{1}, x_{i, g}^{2}, \ldots, x_{i, g}^{d}\right\}$ is the $\mathrm{i}^{\text {th }}$ solution (individual) of the population, $P=\left\{X_{1}, X_{2}, \ldots, X_{N P}\right\}$ at the $\mathrm{g}^{\text {th }}$ iteration where $\mathrm{d}$ is the data dimensionality and NP is the size of population.

\subsubsection{Mutation}

A trial vector $V_{i, g}$ is generated for each parent solution $X_{i, g}$ by perturbing a target solution, $X_{i 1, g}$ with a scaled difference as follows:

$$
V_{i, g}=X_{i 1, g}+f\left(X_{i 2, g}-X_{i 3, g}\right)
$$

Where $i$ is an integer within [1, NP] $i_{1}, i_{2}$, and $i_{3}$ are random integers within [1, NP] such that $i \neq i 1 \neq$ $i 2 \neq i 3$, and then $f$ is a scaled factor within $(0, \infty)$.

\subsubsection{Crossover}

At the crossover phase, an offspring vector, $U_{i, g}$ is usually generated by applying binomial crossover operator as follows:

$$
u_{i, g}^{j}= \begin{cases}v_{i, g}^{j} & \text { if } \operatorname{rand}(j) \leq C R \\ x_{i, g}^{j} & \text { otherwise }\end{cases}
$$

Where $i$ an integers within $[1, \mathrm{NP}], j$ is an integer within $[1, \mathrm{~d}], \operatorname{rand}(j) \in \mathrm{U}(0,1)$ and the crossover rate, $C R \in$ $(0,1)$.

\subsubsection{Selection}

In the selection phase, the parent solution in the current population and its offspring vector are compared to determine which will remain in the next generation (iteration). The fitter solution is selected and added to the new population. For the maximizing problem, the solution vector for the next iteration is chosen according to the following;

$$
X_{i, g+1}= \begin{cases}U_{i, g} & \text { if } f\left(U_{i, g}\right)>f\left(X_{i, g}\right) \\ X_{i, g} & \text { otherwise }\end{cases}
$$

Where $f\left(U_{i, g}\right)$ indicates the fitness value of offspring and $f\left(X_{i, g}\right)$ denotes the fitness value of $\mathrm{i}^{\text {th }}$ parent in the current population.

\subsection{Different Mutation Strategies in Differential Evolution}

Recent research works proposed numerous variants to the basic DE. In the literature, a notation of $\mathrm{DE} / \mathrm{x} / \mathrm{y} / \mathrm{z}$ is commonly used to categorize these different variants [1]. In this notation, $\mathrm{x}$ indicates the way of choosing a target solution, $y$ specifies the number of pairs of difference vectors applied, and the last symbol, $\mathrm{z}$, identifies the adopted crossover operator. This paper intends to describe various mutation strategies. Thus, the notation DE/x/y is applied, and the character $\mathrm{z}$ is omitted. The random mutation strategy, DE/rand/1 is typically used in a standard DE algorithm. The mutation strategies [2, 3] that are frequently used are as follows. 


\subsubsection{Random Mutation Strategy}

$\mathrm{DE} / \mathrm{rand} / 1$ and $\mathrm{DE} / \mathrm{rand} / 2$ are the random mutation strategies that use one difference vector and two difference vectors, respectively. As mentioned in above, $\mathrm{DE} / \mathrm{rand} / 1$ creates the trial vector with three randomly chosen solution vectors while $\mathrm{DE} / \mathrm{rand} / 2$ uses five randomly selected solution vectors to generate the trial vector according to the following equation;

$$
V_{i, g}=X_{i 1, g}+f_{1}\left(X_{i 2, g}-X_{i 3, g}\right)+f_{2}\left(X_{i 4, g}-X_{i 5, g}\right)
$$

Where $f_{1}$ and $f_{2}$ are two control parameters to scale differences of vectors, il, i2, i3, i4, and $i 5$ are disjoint randomly generated integers within $[1, \mathrm{NP}]$.

\subsubsection{Best Mutation Strategy}

The best mutation strategy applies the fittest solution vector in the population as the target vector. DE/best/1 and DE/best/2 represent two types of the best mutation strategy that use one difference vector and two difference vectors, respectively. These strategies generate the trial vectors as follows;

$$
\begin{aligned}
& V_{i, g}=X_{\text {best }, g}+f\left(X_{i 1, g}-X_{i 2, g}\right) \\
& V_{i, g}=X_{\text {best }, g}+f_{1}\left(X_{i 1, g}-X_{i 2, g}\right)+f_{2}\left(X_{i 3, g}-X_{i 4, g}\right)
\end{aligned}
$$

Where $f, f_{1}$ and $f_{2}$ are the scaling factors within $(0, \infty)$, and $i 1, i 2, i 3$, and $i 4$ are disjoint randomly generated integers within $[1, \mathrm{NP}]$.

\subsubsection{Current to Random Mutation Strategy}

The notation DE/current-to-rand/1 indicates the current to random mutation strategy. This strategy uses a parent solution as a target vector and employed two difference vectors to produce a trial vector. The first difference is the difference between one random solution and the parent solution, whereas the rest is computed from two randomly selected vectors. DE/current-to-rand/1 produces the trial vector according to the following equation;

$$
V_{i, g}=X_{i, g}+f_{1}\left(X_{\mathrm{i} 1, g}-X_{i, g}\right)+f_{2}\left(X_{i 2, g}-X_{i 3, g}\right)
$$

Where $f_{1}$ and $f_{2} \in(0, \infty)$ are the scaling factors, and $i 1, i 2$ and $i 3$ are different randomly generated indexes within $[1, \mathrm{NP}]$.

\subsubsection{Current to Best Mutation Strategy}

This strategy is also known as the target to best mutation strategy and represented by the notation $\mathrm{DE} /$ current-to-best/1. It uses the parent solution as a target vector and applies two difference vectors to mutate the target vector. The first difference vector is calculated from the best and parent solutions, whereas the rest is computed from two randomly selected vectors. The trial vector is produced as followings;

$$
V_{i, g}=X_{i, g}+f_{1}\left(X_{\text {best }, g}-X_{i, g}\right)+f_{2}\left(X_{i 1, g}-X_{i 2, g}\right)
$$

Where $f_{1}$ and $f_{2} \in(0, \infty)$ are the scale number for controlling difference vector, and $i 1$ and $i 2 \in[1, \mathrm{NP}]$ that are different randomly generated indexes.

\section{DIFFERENTIAL EVOLUTION BASED CLUSTERING ALGORITHM}

DE maintains a number of possible solutions to the problem as a population. Each possible solution is encoded as a chromosome (individual). For applying DE to solve clustering problems, a cluster solution for the given data set is encoded as an individual. And then, cluster validity measures are used as objective functions to evaluate the fitness of the solution [11].

In this paper, centroid-based representation is used where a chromosome is encoded by real numbers, which represents the coordinates of centroids of a cluster solution. If a chromosome encodes $\mathrm{k}$ clusters of a ddimensional dataset, $\mathrm{k} * \mathrm{~d}$ is the length of this chromosome. Each chromosome of the initial population is constructed as follows; $X_{i}=\left\{x_{i}^{1}, x_{i}^{2}, \ldots, x_{i}^{d}, x_{i}^{d+1}, x_{i}^{d+2}, \ldots, x_{i}^{2 d}, \ldots, x_{i}^{(k-1) d+1}, x_{i}^{(k-1) d+2}, \ldots, x_{i}^{k d}\right\}$ where the very first d-dimensional vector stands for the first cluster centroid, the second d-dimensional vector denotes the coordinate of the second cluster center, and the last $\mathrm{d}$-dimensional vector represent the $\mathrm{k}^{\text {th }}$ cluster centroid for the given data set. The total intra-cluster distance [7] is used as an objective function to compute the fitness of each chromosome.

$$
\text { TotalInraClusterDistance }=\sum_{j=1}^{k} \sum_{d \in C_{j}} \operatorname{Dist}\left(d, c_{j}\right)
$$

Where k is the number of cluster, $C_{j}$ is the jth cluster, $\mathrm{d}$ is a data point in $C_{j}, c_{j}$ is the center of $C_{j}$, and Dist is the Euclidean distance [12] between data point $\mathrm{d}$ and the center $c_{j}$ of the cluster $C_{j}$.

In DE based clustering algorithm, each chromosome is initialized with $\mathrm{k}$ randomly selected cluster centers from a given dataset to construct an initial population. To compute the fitness of each chromosome, Euclidean distance between each data point and all cluster center of the chromosome is firstly calculated, and then the data points are assigned to the closet cluster, and finally, the sum of intra-cluster distance of each 
cluster is calculated. The population for the next generation is produced by mutation, crossover and selection. The best solution of the final population is the optimal cluster solution for the given dataset. The process of DE based clustering algorithm is given in Algorithm 1.

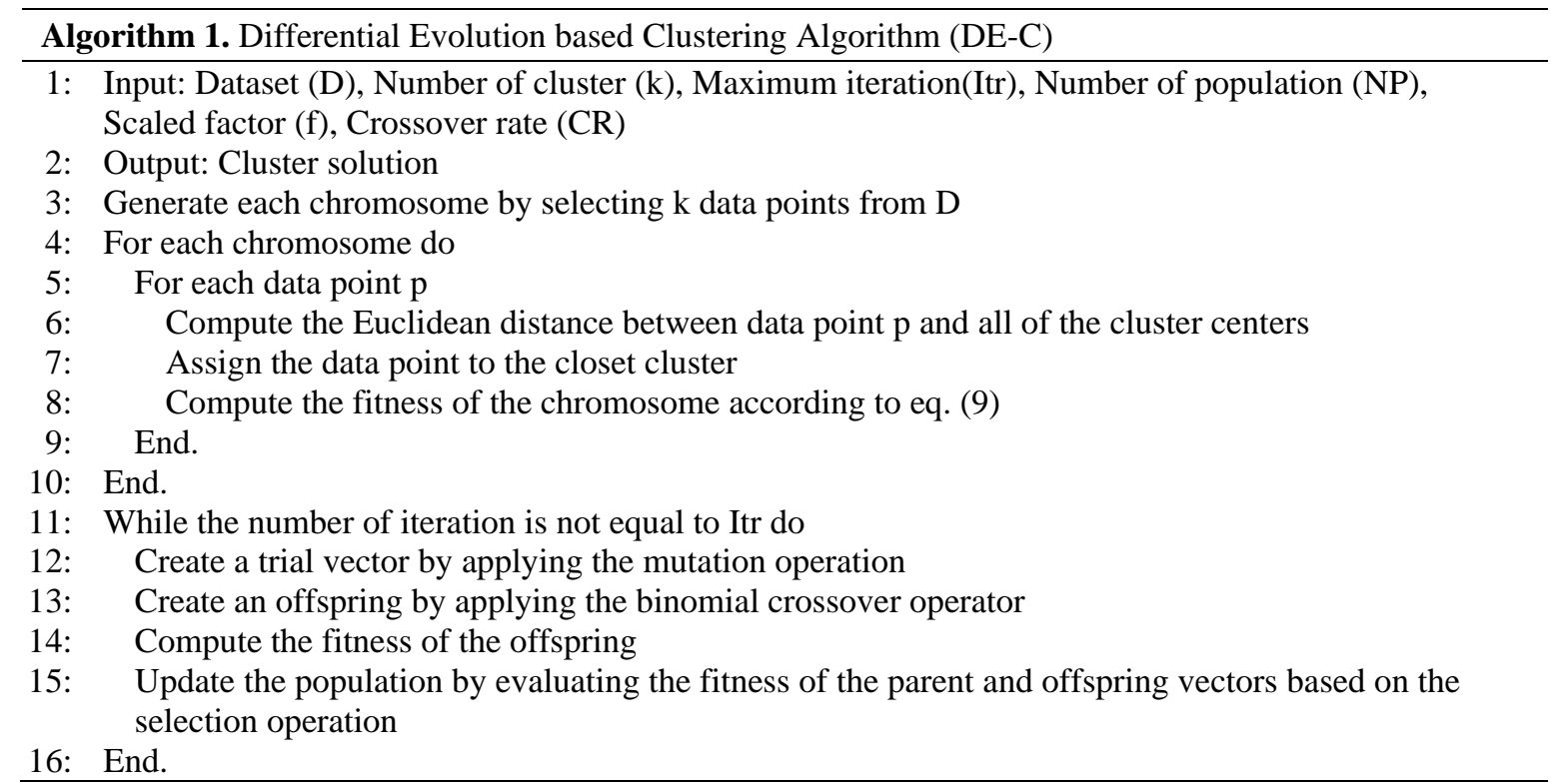

\section{EXPERIMENTAL STUDY}

The main aim of this work is to provide some valuable information for developing a simple, efficient and robust DE based clustering algorithm. The most well-known, simple and efficient mutation strategies were taken into account in this work. The clustering performance of DE algorithms with six different mutation strategies is tested on some real datasets from the UCI machine learning repositroy [13]. Seven UCI standard datasets that are frequently used for metahuristics-based clustering [14] are utilized. The summary of these datasets is shown in Table 1.

\subsection{Experimental Setup}

For all DE based clustering algorithms with different mutation strategies, the crossover rate and the size of the population were respectively set to 0.9 and 100 [7, 15], and the scaling factor was set as follows: $f=0.5, f_{l}=0.3$ and $f_{2}=0.3$. The number of maximum iteration was set to 100 . The initial population was constructed in a similar fashion such that each chromosome was composed of $\mathrm{k}$ distinct data points that were randomly selected from the dataset. The algorithms were implemented in java programming language on Intel Core i7 processor, 8GB memory, and 64-bit operating system. Each algorithm was executed 30 times independently for each dataset.

Table 1. Summary of the used datasets

\begin{tabular}{cccc}
\hline Datasets & No. of Attributes & No. of Instances & No. of Cluster \\
\hline Iris (Iris plant data) & 4 & 150 & 3 \\
Wine (Wine recognition data) & 13 & 178 & 3 \\
Thyroid (Thyroid Disease Data) & 5 & 215 & 3 \\
Breast Cancer (Wisconsin Diagnostic & 9 & 683 & 2 \\
Breast Cancer Data) & 18 & 768 & 2 \\
Pima (Pima Indians Diabetes Data) & 10 & 214 & 6 \\
Glass (Glass Identification Data) & 8 & 336 & 8 \\
Ecoli (Protein Localization Sites) & & & \\
\hline
\end{tabular}

The quality of obtained cluster solutions and convergence speed of different DE variants were compared. The quality of clustering solutions was compared according to the following criteria:

- The objective function values (total intra-cluster distance defined in eq. (9) )

- Sum of squared error (SSE) [12]: It calculate the sum of the squared distances from each data point in a cluster to the center of this cluster as follows: 
$S S E=\sum_{j=1}^{k} \sum_{d \in C_{j}} \operatorname{Dist}\left(d, c_{j}\right)^{2}$

Where k represents the number of clusters, $C_{j}$ stands for the jth cluster, $\mathrm{d}$ is a data point in $C_{j}, c_{j}$ is the center of $C_{j}$, and Dist is the Euclidean distance between data point $\mathrm{d}$ and the center $c_{j}$ of the cluster $C_{j}$. Minimum SSE indicates better cluster solutions.

Table 2. Comparison of objective function values

\begin{tabular}{|c|c|c|c|c|c|}
\hline Dataset & Mutation Strategy & Worst & Best & Mean & Std \\
\hline \multirow[t]{6}{*}{ Iris } & $\mathrm{DE} / \mathrm{rand} / 1$ & 97.91498 & 96.91073 & 97.3809244 & 0.286711212 \\
\hline & $\mathrm{DE} / \mathrm{rand} / 2$ & 104.59639 & 100.58503 & 102.1337669 & 1.18710493 \\
\hline & DE/best/1 & 97.66495 & 96.70644 & 97.1257265 & 0.287769934 \\
\hline & DE/best/2 & 96.688 & 96.6557 & 96.6670415 & 0.010426543 \\
\hline & DE/current-to-rand/1 & 98.56196 & 97.7142 & 98.2257235 & 0.2330539 \\
\hline & DE/current-to-best/1 & 96.85357 & 96.676285 & 96.7280826 & 0.067527291 \\
\hline \multirow[t]{6}{*}{ Wine } & $\mathrm{DE} / \mathrm{rand} / 1$ & 16296.588 & 16291.879 & 16293.9099 & 1.798194616 \\
\hline & $\mathrm{DE} / \mathrm{rand} / 2$ & 16424.855 & 16352.439 & 16386.8182 & 24.68133014 \\
\hline & DE/best/1 & 16324.859 & 16310.749 & 16316.9338 & 5.232458479 \\
\hline & $\mathrm{DE} / \mathrm{best} / 2$ & 16309.574 & 16295.889 & 16300.9111 & 4.21486466 \\
\hline & DE/current-to-rand/1 & 16315.1 & 16300.865 & 16306.5729 & 4.953413693 \\
\hline & DE/current-to-best/1 & 16299.441 & 16294.082 & 16296.246 & 1.755794977 \\
\hline \multirow[t]{6}{*}{ Thyroid } & $\mathrm{DE} / \mathrm{rand} / 1$ & 1882.7504 & 1866.4769 & 1869.45039 & 4.93738597 \\
\hline & $\mathrm{DE} / \mathrm{rand} / 2$ & 1933.1353 & 1904.8689 & 1922.52497 & 8.792795624 \\
\hline & DE/best/1 & 1892.2047 & 1879.5593 & 1883.38591 & 3.564562431 \\
\hline & DE/best $/ 2$ & 1876.3129 & 1866.6946 & 1871.08952 & 2.978686652 \\
\hline & DE/current-to-rand/1 & 1896.8396 & 1877.1492 & 1888.41288 & 5.396856437 \\
\hline & DE/current-to-best/1 & 1884.3173 & 1867.5745 & 1874.91641 & 4.83995231 \\
\hline \multirow[t]{6}{*}{ Breast Cancer } & $\mathrm{DE} / \mathrm{rand} / 1$ & 2971.8862 & 2964.4321 & 2966.53782 & 2.650869494 \\
\hline & $\mathrm{DE} / \mathrm{rand} / 2$ & 3311.5127 & 2965.7437 & 3012.85072 & 105.5592452 \\
\hline & $\mathrm{DE} /$ best/1 & 3033.665 & 3000.5054 & 3018.80216 & 11.39918918 \\
\hline & DE/best/2 & 2977.0986 & 2966.4019 & 2971.18367 & 4.13411904 \\
\hline & DE/current-to-rand/1 & 2999.9495 & 2973.002 & 2987.84142 & 8.637445694 \\
\hline & $\mathrm{DE} /$ current-to-best/1 & 2974.674 & 2964.858 & 2969.47028 & 3.284382688 \\
\hline \multirow[t]{6}{*}{ Pima } & $\mathrm{DE} / \mathrm{rand} / 1$ & 47569.33 & 47561.23 & 47563.2065 & 3.092244609 \\
\hline & $\mathrm{DE} / \mathrm{rand} / 2$ & 47678.477 & 47564.79 & 47601.2339 & 38.04236253 \\
\hline & DE/best/1 & 47975.336 & 47780.234 & 47884.8688 & 54.80166752 \\
\hline & DE/best/2 & 47591.04 & 47562.61 & 47575.7867 & 10.42867773 \\
\hline & DE/current-to-rand/1 & 47614.9 & 47563.367 & 47587.5049 & 17.31487431 \\
\hline & DE/current-to-best/1 & 47572.508 & 47563.207 & 47566.7832 & 2.977584547 \\
\hline \multirow[t]{6}{*}{ Glass } & $\mathrm{DE} / \mathrm{rand} / 1$ & 238.61061 & 214.88739 & 224.339602 & 7.936183946 \\
\hline & $\mathrm{DE} / \mathrm{rand} / 2$ & 256.1896 & 239.43997 & 246.430862 & 4.351225392 \\
\hline & DE/best/1 & 233.55023 & 226.03458 & 229.798054 & 2.584396108 \\
\hline & $\mathrm{DE} / \mathrm{best} / 2$ & 223.81511 & 217.97005 & 221.988005 & 1.830325338 \\
\hline & DE/current-to-rand/1 & 246.86244 & 241.1548 & 244.030698 & 2.155017781 \\
\hline & DE/current-to-best/1 & 230.9455 & 218.51709 & 222.542509 & 4.262207958 \\
\hline \multirow[t]{6}{*}{ Ecoli } & $\mathrm{DE} / \mathrm{rand} / 1$ & 70.950424 & 64.20288 & 67.583721 & 2.17513627 \\
\hline & $\mathrm{DE} / \mathrm{rand} / 2$ & 74.87054 & 71.46696 & 73.1026902 & 1.238720257 \\
\hline & DE/best/1 & 69.42949 & 66.73027 & 67.9053545 & 0.904321255 \\
\hline & $\mathrm{DE} / \mathrm{best} / 2$ & 66.2538 & 64.51221 & 65.3631044 & 0.628261569 \\
\hline & DE/current-to-rand/1 & 73.64982 & 71.09162 & 72.1286431 & 0.66660349 \\
\hline & $\mathrm{DE} /$ current-to-best/1 & 69.25693 & 65.226425 & 66.9274105 & 1.263528514 \\
\hline
\end{tabular}

- Quantization error [16]: It calculates the average distance between data points and the cluster center as follows:

$$
J_{e}=\frac{\sum_{j}^{k}\left[\sum_{\forall d \in C_{j}} \operatorname{Dist}\left(d, c_{j}\right) /\left|C_{j}\right|\right]}{k}
$$

Where k indicate the number of clusters, $C_{j}$ stands for the jth cluster, $\mathrm{d}$ is a data point in $C_{j}, c_{j}$ is the center of $C_{j},\left|C_{j}\right|$ is the number of data points in $\mathrm{Cj}$ and Dist is the Euclidean distance between data point $\mathrm{d}$ and the center $c_{j}$ of the cluster $C_{j}$. Lower quantization means the better cluster results.

\subsection{Experimental Results}

The experimental results obtained by DE based clustering algorithms with different mutation strategies are summarized in Table 2-4. The qualities of solutions obtained by each algorithm are tabulated in terms of the worst, best, mean and standard deviation (Std.).

The vales of objective function obtained for all datasets are presented in Table 2. According to the mean values of the given results in Table 2, DE/rand/1 got better results for four datasets while DE/best $/ 2$ got better solutions for Iris and other two datasets with high number of clusters (Glass and Ecoli). The values of standard deviation obtained by DE/best/2 are smaller than these values got by other variants for Iris, Thyroid, 
Glass, and Ecoli datasets. For Wine, Breast Cancer, and Pima datasets, the values of standard deviation achieved by DE/current-to-best/1 are smaller than the values acquired by others. It can be said that DE/best/2 and $\mathrm{DE} /$ current-to-best/1 is more robust than others where DE/current-to-best/1acheived more stable results for high-dimensional datasets (in terms of number of feature and number of data instances) than DE/best/2.

Table 3. Comparison of sum of squared error

\begin{tabular}{|c|c|c|c|c|c|}
\hline Dataset & Mutation Strategy & Worst & Best & Mean & Std \\
\hline \multirow[t]{6}{*}{ Iris } & $\mathrm{DE} / \mathrm{rand} / 1$ & 83.629715 & 80.42072 & 82.0800965 & 0.94004685 \\
\hline & $\mathrm{DE} / \mathrm{rand} / 2$ & 89.5296 & 84.717865 & 87.5544574 & 1.842881411 \\
\hline & DE/best/1 & 81.711655 & 80.14506 & 80.9866801 & 0.654303667 \\
\hline & $\mathrm{DE} /$ best $/ 2$ & 80.38089 & 79.99243 & 80.1618585 & 0.113351464 \\
\hline & DE/current-to-rand/1 & 85.98539 & 81.2372 & 83.3685664 & 1.488298053 \\
\hline & DE/current-to-best/1 & 80.505196 & 80.0851 & 80.3149833 & 0.154577131 \\
\hline \multirow[t]{6}{*}{ Wine } & $\mathrm{DE} / \mathrm{rand} / 1$ & 2594709 & 2513174.5 & 2564749 & 27068.79947 \\
\hline & $\mathrm{DE} / \mathrm{rand} / 2$ & 2655556 & 2447204.2 & 2537712.62 & 70151.07796 \\
\hline & DE/best/1 & 2611303 & 2506935 & 2549237.9 & 31776.9966 \\
\hline & $\mathrm{DE} /$ best $/ 2$ & 2595641.8 & 2510130.2 & 2552362.65 & 27419.28448 \\
\hline & DE/current-to-rand/1 & 2624745.8 & 2515277.8 & 2580781.38 & 32488.16905 \\
\hline & DE/current-to-best/1 & 2599812.5 & 2542793.5 & 2581293.87 & 21449.25797 \\
\hline \multirow[t]{6}{*}{ Thyroid } & $\mathrm{DE} / \mathrm{rand} / 1$ & 35247.93 & 34424.89 & 34884.4144 & 239.2168449 \\
\hline & $\mathrm{DE} / \mathrm{rand} / 2$ & 36917.297 & 32058.793 & 35135.2568 & 1563.933731 \\
\hline & $\mathrm{DE} / \mathrm{best} / 1$ & 36179.496 & 34523.406 & 35391.341 & 533.1252703 \\
\hline & $\mathrm{DE} /$ best $/ 2$ & 35384.438 & 34568.34 & 35073.3447 & 272.6169406 \\
\hline & DE/current-to-rand/1 & 36213.64 & 34646.14 & 35288.382 & 537.5361905 \\
\hline & DE/current-to-best/1 & 35627.25 & 34905.703 & 35201.0054 & 237.0561105 \\
\hline \multirow[t]{6}{*}{ Breast Cancer } & $\mathrm{DE} / \mathrm{rand} / 1$ & 19609.496 & 19444.648 & 19503.1858 & 58.33649301 \\
\hline & $\mathrm{DE} / \mathrm{rand} / 2$ & 22647.238 & 19426.004 & 19922.5529 & 973.8623906 \\
\hline & DE/best/1 & 20586.848 & 19734.09 & 20163.7867 & 271.2584436 \\
\hline & DE/best/2 & 19747.975 & 19435.182 & 19558.3365 & 103.611039 \\
\hline & DE/current-to-rand/1 & 20101.18 & 19639.258 & 19871.8596 & 169.3518486 \\
\hline & DE/current-to-best/1 & 19715.303 & 19453.16 & 19568.6758 & 84.81113703 \\
\hline \multirow[t]{6}{*}{ Pima } & $\mathrm{DE} / \mathrm{rand} / 1$ & 5878486.5 & 5873893.5 & 5876531.25 & 1418.108702 \\
\hline & $\mathrm{DE} / \mathrm{rand} / 2$ & 5942486 & 5849599.5 & 5895572.6 & 25906.31329 \\
\hline & DE/best/1 & 6024383 & 5822446 & 5909666.1 & 57765.50274 \\
\hline & DE/best/2 & 5901308.5 & 5873936.5 & 5882881.75 & 8154.457415 \\
\hline & DE/current-to-rand/1 & 5966982 & 5869100.5 & 5916646.85 & 28769.17591 \\
\hline & DE/current-to-best/1 & 5882846 & 5872621.5 & 5878205.2 & 2936.693797 \\
\hline \multirow[t]{6}{*}{ Glass } & $\mathrm{DE} / \mathrm{rand} / 1$ & 517.1698 & 352.20148 & 451.584795 & 51.7028711 \\
\hline & $\mathrm{DE} / \mathrm{rand} / 2$ & 620.7019 & 490.95703 & 554.339182 & 39.59907819 \\
\hline & DE/best/1 & 534.244 & 470.30612 & 504.460414 & 21.25916904 \\
\hline & $\mathrm{DE} / \mathrm{best} / 2$ & 511.1626 & 449.67932 & 479.885252 & 18.79054734 \\
\hline & DE/current-to-rand/1 & 588.00256 & 462.1183 & 521.978414 & 34.68964865 \\
\hline & DE/current-to-best/1 & 506.72964 & 400.1598 & 467.785169 & 32.75475979 \\
\hline \multirow[t]{6}{*}{ Ecoli } & $\mathrm{DE} / \mathrm{rand} / 1$ & 18.250305 & 15.541359 & 17.0615069 & 1.064179566 \\
\hline & $\mathrm{DE} / \mathrm{rand} / 2$ & 21.400398 & 19.9141 & 20.3151097 & 0.483599322 \\
\hline & DE/best/1 & 18.451756 & 15.876751 & 17.5507899 & 0.736882932 \\
\hline & DE/best $/ 2$ & 16.891884 & 15.218651 & 16.3252521 & 0.569443113 \\
\hline & DE/current-to-rand/1 & 20.323498 & 18.774597 & 19.4565839 & 0.552628073 \\
\hline & DE/current-to-best/1 & 18.026596 & 16.368816 & 17.2151671 & 0.597203844 \\
\hline
\end{tabular}

Table 3 and Table 4 summarized the quality of cluster solutions acquired from different mutation strategies in terms of the sum of squared error (SSE) and quantization error, respectively. According to the mean values given in both Table 3 and Table 4, the solutions acquired by random mutation strategies (DE/rand/1 and DE/rand/2) are better than other strategies for almost all of the test datasets. However, the mutation strategies that involve the best vector (DE/best/1, DE/best/2 and DE/current-to-best/1) obtained more stable results than others according to the standard deviation values given in both tables.

The convergence manners of different mutation strategies for all of the test datasets are shown in Figure 1. Based on the same 30 separate runs as mentioned above, the figure is illustrated with the averages of this runs. As observed in Figure 1, the convergence speed of DE/best/2 is the fastest on all datasets, whereas $\mathrm{DE} / \mathrm{rand} / 2$ is the slowest and worst mutation strategy for all test datasets. Although DE/best/1 is faster than all variants except $\mathrm{DE} / \mathrm{best} / 2$, it is not able to search for better solutions in the later stages, and it easily catches to local optima. DE/rand/1 finds better solutions for some datasets than other strategies in the late iterations, even though its convergence rate is slow in the early stages. DE/current-to-rand/1 can be regarded as a second-worst mutation strategy because it is slower and does not reach a better solution for all datasets except Iris. The exploration ability of DE/current-to-best/1 is not sufficient, and it does not catch a better solution for some datasets, although its convergence speed is a little fast. 
Table 4. Comparison of quantization error

\begin{tabular}{|c|c|c|c|c|c|}
\hline Dataset & Mutation Strategy & Worst & Best & Mean & Std \\
\hline \multirow[t]{6}{*}{ Iris } & $\mathrm{DE} / \mathrm{rand} / 1$ & 0.6516792 & 0.644194 & 0.648230088 & 0.002096196 \\
\hline & $\mathrm{DE} / \mathrm{rand} / 2$ & 0.6917781 & 0.6613507 & 0.678133994 & 0.009085603 \\
\hline & DE/best/1 & 0.6529307 & 0.6434465 & 0.647316521 & 0.003001606 \\
\hline & $\mathrm{DE} / \mathrm{best} / 2$ & 0.64522 & 0.6437538 & 0.643967262 & 0.00044599 \\
\hline & DE/current-to-rand/1 & 0.6572807 & 0.6491747 & 0.654163175 & 0.002575488 \\
\hline & DE/current-to-best/1 & 0.6464462 & 0.6439006 & 0.644522413 & 0.00101929 \\
\hline \multirow[t]{6}{*}{ Wine } & $\mathrm{DE} / \mathrm{rand} / 1$ & 96.143974 & 95.56788 & 95.7610459 & 0.185679641 \\
\hline & $\mathrm{DE} / \mathrm{rand} / 2$ & 97.008766 & 96.04605 & 96.4994756 & 0.295641799 \\
\hline & DE/best/1 & 96.29065 & 95.70694 & 96.0187704 & 0.211108131 \\
\hline & DE/best/2 & 96.216225 & 95.6381 & 95.8906245 & 0.181166983 \\
\hline & DE/current-to-rand/1 & 96.16913 & 95.63155 & 95.8116934 & 0.182229921 \\
\hline & DE/current-to-best/1 & 95.86396 & 95.57987 & 95.668115 & 0.12394908 \\
\hline \multirow[t]{6}{*}{ Thyroid } & $\mathrm{DE} / \mathrm{rand} / 1$ & 9.302322 & 8.971374 & 9.09637795 & 0.115034208 \\
\hline & $\mathrm{DE} / \mathrm{rand} / 2$ & 11.713471 & 9.0082445 & 9.77102625 & 0.735706345 \\
\hline & DE/best/1 & 9.33271 & 8.854203 & 9.15417135 & 0.161775128 \\
\hline & DE/best/2 & 9.221522 & 9.0455675 & 9.14978185 & 0.064162164 \\
\hline & DE/current-to-rand/1 & 9.469611 & 9.058529 & 9.2730773 & 0.107299489 \\
\hline & DE/current-to-best/1 & 9.312383 & 8.98903 & 9.1844789 & 0.083235219 \\
\hline \multirow[t]{6}{*}{ Breast Cancer } & $\mathrm{DE} / \mathrm{rand} / 1$ & 5.21077 & 5.1888194 & 5.19391174 & 0.006918966 \\
\hline & $\mathrm{DE} / \mathrm{rand} / 2$ & 5.765476 & 5.191242 & 5.272178 & 0.174804199 \\
\hline & DE/best/1 & 5.351944 & 5.2500167 & 5.29663055 & 0.031849959 \\
\hline & $\mathrm{DE} /$ best $/ 2$ & 5.2154016 & 5.192358 & 5.20259006 & 0.00856287 \\
\hline & DE/current-to-rand/1 & 5.2407265 & 5.20192 & 5.22435062 & 0.012082721 \\
\hline & DE/current-to-best/1 & 5.2095737 & 5.189504 & 5.19876374 & 0.007111739 \\
\hline \multirow[t]{6}{*}{ Pima } & $\mathrm{DE} / \mathrm{rand} / 1$ & 67.81502 & 67.800385 & 67.803803 & 0.005570987 \\
\hline & $\mathrm{DE} / \mathrm{rand} / 2$ & 67.93898 & 67.770996 & 67.8345055 & 0.051236144 \\
\hline & DE/best/1 & 68.52382 & 67.7503 & 68.247306 & 0.221579355 \\
\hline & $\mathrm{DE} /$ best $/ 2$ & 67.8479 & 67.802315 & 67.822286 & 0.01679569 \\
\hline & DE/current-to-rand/1 & 67.82375 & 67.614174 & 67.7693949 & 0.067872086 \\
\hline & DE/current-to-best/1 & 67.81558 & 67.802895 & 67.8087031 & 0.004526859 \\
\hline \multirow[t]{6}{*}{ Glass } & $\mathrm{DE} / \mathrm{rand} / 1$ & 1.7753247 & 1.1713182 & 1.42850018 & 0.216891528 \\
\hline & $\mathrm{DE} / \mathrm{rand} / 2$ & 1.7360297 & 1.1389076 & 1.459802 & 0.189753391 \\
\hline & DE/best/1 & 1.6024362 & 1.2440042 & 1.36619334 & 0.120531122 \\
\hline & $\mathrm{DE} /$ best $/ 2$ & 1.5341662 & 1.1582086 & 1.32218297 & 0.097018546 \\
\hline & DE/current-to-rand/1 & 1.8478018 & 1.2105691 & 1.5327666 & 0.193121642 \\
\hline & $\mathrm{DE} /$ current-to-best/1 & 1.6957501 & 1.2079638 & 1.38235998 & 0.156474922 \\
\hline \multirow[t]{6}{*}{ Ecoli } & $\mathrm{DE} / \mathrm{rand} / 1$ & 0.2327994 & 0.1982598 & 0.212012397 & 0.010604917 \\
\hline & DE/rand/2 & 0.2355672 & 0.2026296 & 0.221384451 & 0.008893089 \\
\hline & DE/best/1 & 0.2153705 & 0.1989665 & 0.205757483 & 0.004651351 \\
\hline & $\mathrm{DE} / \mathrm{best} / 2$ & 0.2163881 & 0.1932458 & 0.203574999 & 0.008118651 \\
\hline & DE/current-to-rand/1 & 0.2319987 & 0.199595 & 0.2186906 & 0.008642931 \\
\hline & DE/current-to-best/1 & 0.2503893 & 0.1883661 & 0.206791102 & 0.020067601 \\
\hline
\end{tabular}

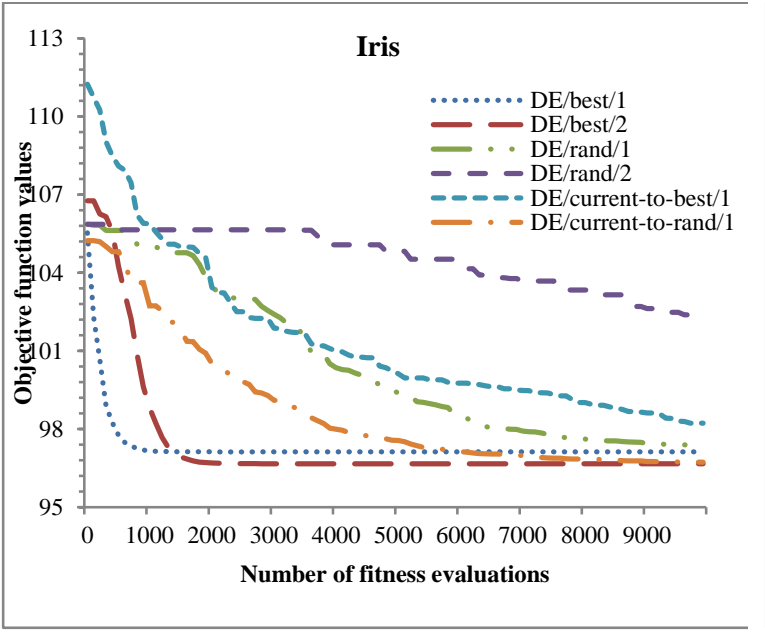

(a)

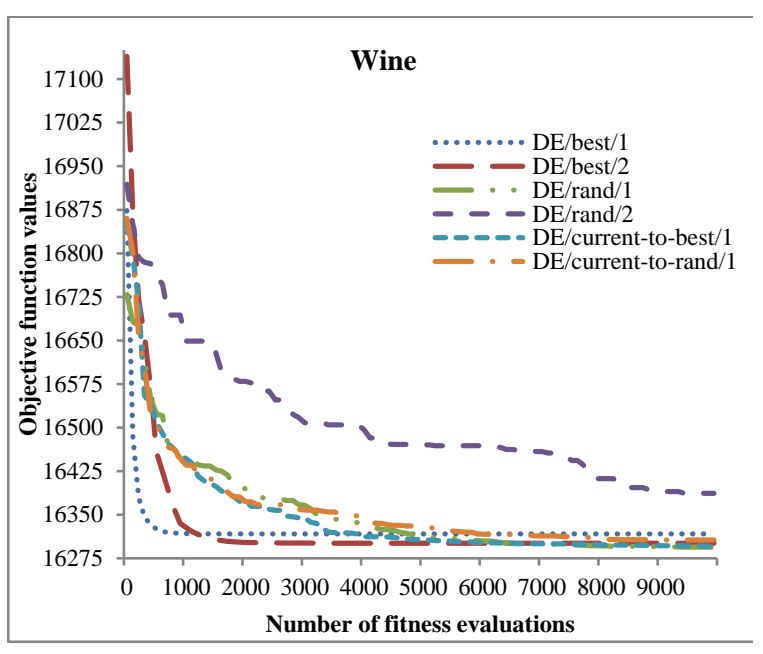

(b) 


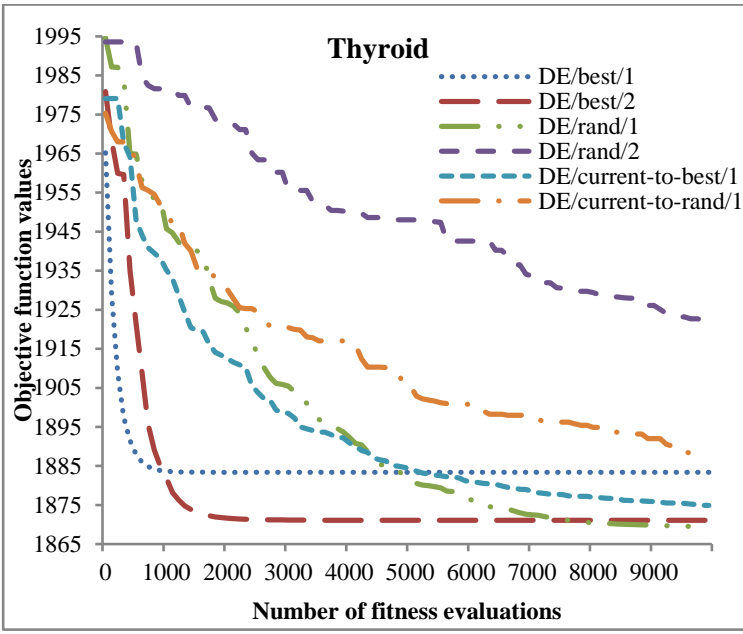

(c)

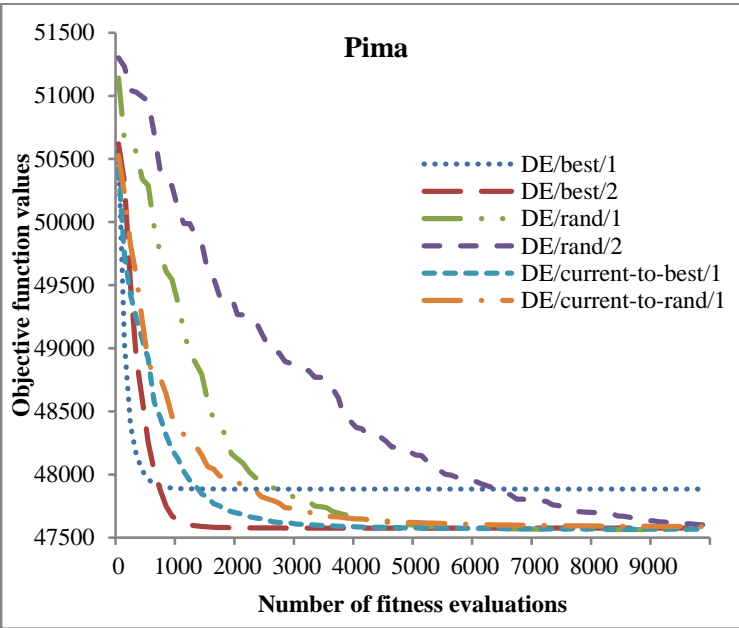

(e)

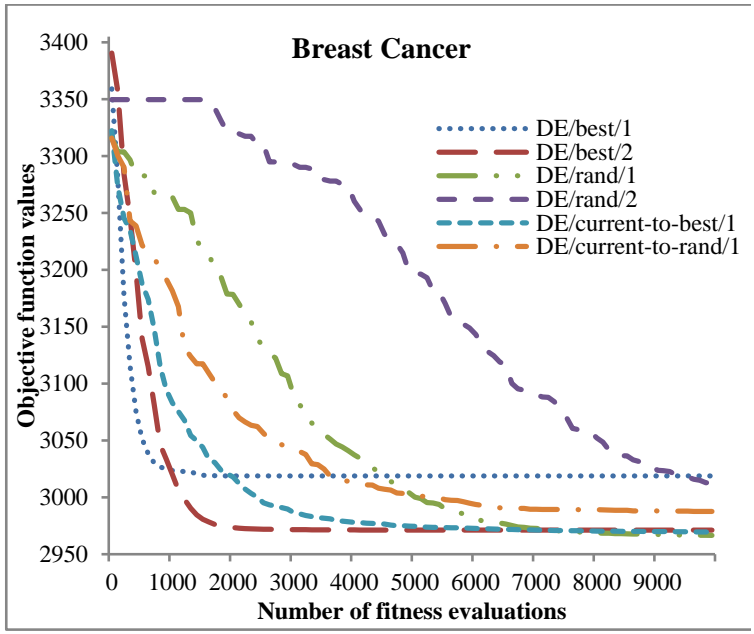

(d)

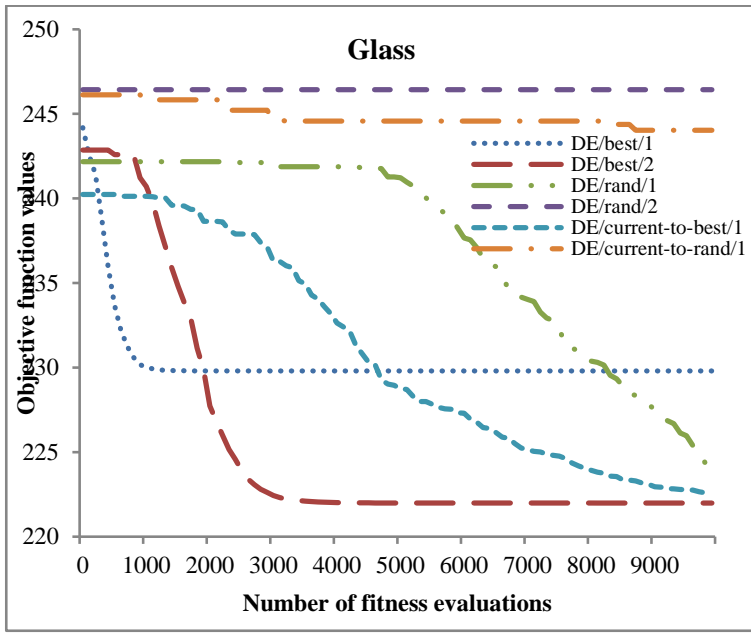

(f)

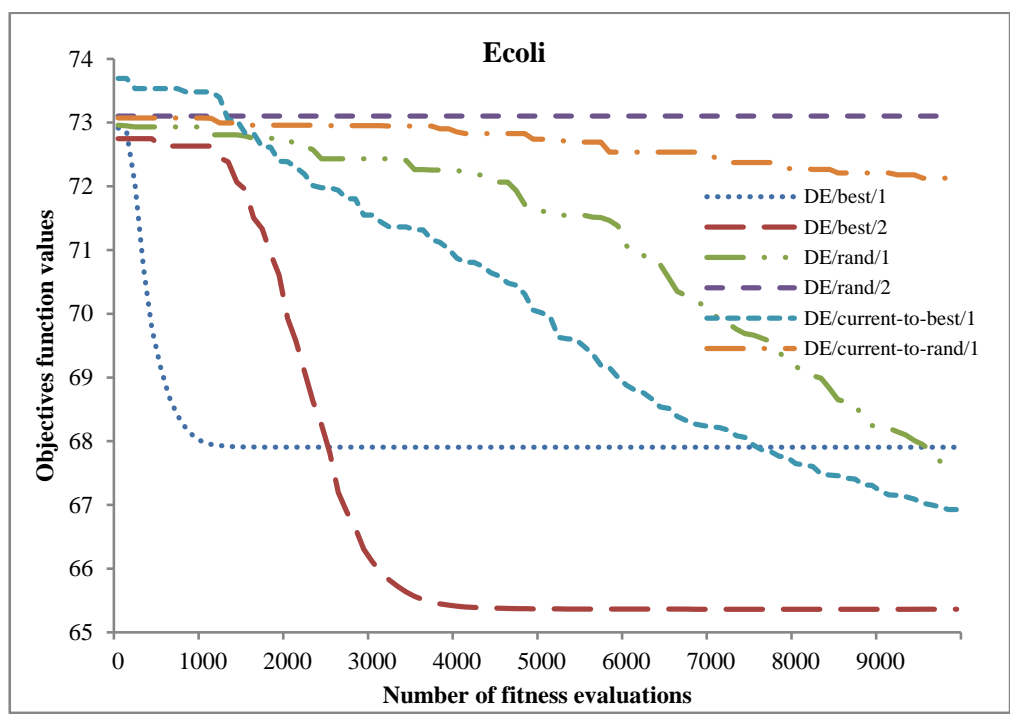

(g)

Figure 1. The convergence performance of different mutation strategies on six datasets 
According to the overall experimental results, it is noticed that as follows: the mutation strategies based on the best solution are more robust and faster than others because these use the guidance information of the best solution to increase the exploitation ability and convergence speed of DE. Among them, DE/best/2 is more effective and robust for datasets with high number of clusters (Glass and Ecoli) due to the guidance information from the best solution and the application of two differentials. DE/rand/1 is able to find better solutions not only for high dimensional datasets (Breast Cancer and Pima) but also for moderate size of datasets because it can keep good diversity.

\section{CONCLUSION}

This paper presents an experimental investigation to analyze different mutation strategies of the DE algorithm for clustering problems. The performance of six mutation strategies has been tested on some UCI standard datasets mostly used in EAs based clustering. The quality of solutions and the convergence speed of different DE variants were compared to investigate the outcomes of the experiments. The experimental analysis pointed out that DE/rand/1 accomplishes to find better solutions for the moderate size of datasets. Besides, it shows good exploitation behavior in the later stages, while DE/best/2 shows good exploration behavior in the early stages. The test also showed that the mutation strategies that used the best solution achieve to find more stable results. Future work is to propose an effective mutation strategy for addressing large-scale clustering problems by applying the information from this experimental study.

\section{REFERENCES}

[1] A. P. Engelbrecht, Computational Intelligence-An Introduction, 2nd ed. England, John Wiley \& Sons, 2007.

[2] S. Das, P. N. Suganthan, "Differential Evolution: A Survey of the State-of-the-Art," IEEE Transactions on Evolutionary Computation, vol. 15, no.1, pp. 4-3, 2010.

[3] R. Storn, and K. Price. "Differential evolution-a simple and efficient heuristic for global optimization over continuous spaces." Journal of global optimization, vol. 11, no. 4, pp. 341-359, 1997.

[4] A. K. Qin, V. L. Huang, and P. N. Suganthan, "Differential evolution algorithm with strategy adaptation for global numerical optimization," IEEE transactions on Evolutionary Computation, vol. 13, no. 2, pp. 398-417, 2008.

[5] S. Paterlini, T. Krink, "High performance clustering with differential evolution," Congress on Evolutionary Computation (CEC2004), pp. 2004-2011, 2004.

[6] S. Paterlini, T. Krink, "Differential evolution and particle swarm optimisation in partitional clustering," Computational Statistics \& Data Analysis, vol. 50, no. 5, pp. 1220-1247, 2006.

[7] W.L. Xiang, N. Zhu, S.F. Ma, , X.L. Meng, and M.Q. An, "A dynamic shuffled differential evolution algorithm for data clustering," Neurocomputing, vol. 158, pp.144-154, 2005.

[8] L. Zhang, L. Mao, H. Gong and H. Yang, "A K-harmonic means clustering algorithm based on enhanced differential evolution," 2013 Fifth International Conference on Measuring Technology and Mechanics Automation, pp. 13-16, 2013.

[9] W. Kwedlo, "A clustering method combining differential evolution with the K-means algorithm," Pattern Recognition Letters, vol. 32, no. 12, pp. 1613-1621, 2011.

[10] L. Mao, H. J.Gong, and X. Y. Liu, "A K-means Clustering Algorithm Based on Enhanced Differential Evolution," in Advanced Materials Research, vol. 339, pp. 71-75, 2011.

[11] E.R. Hruschka, R.J. Campello, and A.A. Freitas, "A survey of evolutionary algorithms for clustering," IEEE Transactions on Systems, Man, and Cybernetics, Part C (Applications and Reviews), vol. 39, no. 2, pp. 133-155, 2009.

[12] A.S. Shirkhorshidi, S. Aghabozorgi, and T.Y. Wah, "A comparison study on similarity and dissimilarity measures in clustering continuous data," PloS one, vol. 10, no. 12, 2015.

[13] https://archive.ics.uci.edu/

[14] S.J. Nanda, and G. Panda, "A survey on nature inspired metaheuristic algorithms for partitional clustering," Swarm and Evolutionary computation, vol. 16, pp. 1-18, 2014.

[15] S. Rahnamayan, H.R. Tizhoosh, and M.M.A. Salama, "Opposition-based differential evolution," IEEE Trans. Evol. Comput., vol. 12, no. 1, pp. 64-79, 2008.

[16] H.T.T. Thein, and K.M.M. Tun, "Evaluation of differential evolution and K-means algorithms on medical diagnosis," in 5th National Symposium on Information Technology: Towards New Smart World (NSITNSW), pp. 1-4, 2015. 


\section{BIOGRAPHY OF AUTHORS}

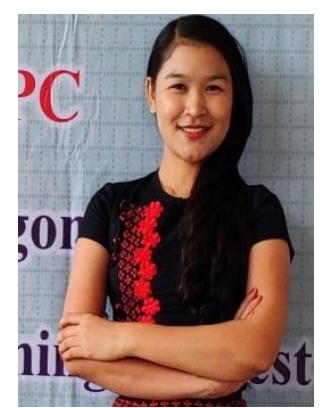

Pyae Pyae Win Cho received the M.C.Sc degree from Computer University, Pathein, Myanmar in 2012. She is an Assistant Lecturer at the University of Computer Studies, Myeik, Myanmar. She is currently working toward a Ph.D. degree at the University of Computer Studies, Yangon, Myanmar. Her research interests include evolutionary computing and data mining.

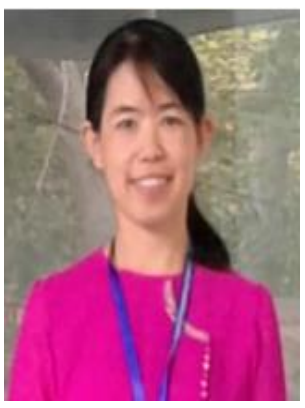

Thi Thi Soe Nyunt got B.Sc. Physics (Hons:) degree from Yangon University in 1994 and got Master of Information Science (M.I.Sc.) degree and Ph. D (IT) from UCSY in 1998 and 2004 respectively. She is currently working as a Professor and Head of department in Faculty of Computer Science, UCSY. Her research interests include Knowledge \& Software Engineering, Database, Computer Graphics, Big Data Analytics, Artificial Intelligence and Neural Network. 